\title{
Placenta accreta spectrum
}

\author{
Valerie Bloomfield MD, Stacey Rogers MD, Nicholas Leyland MD MHCM
}

Cite as: CMAJ 2020 August 24;192:E980. doi: 10.1503/cmaj.200304

\section{Placenta accreta spectrum describes abnormally adherent or \\ 1 invasive placentas}

In normal pregnancy, a blastocyst implants into the endometrium, and after delivery, the placenta detaches from the uterus. In placenta accreta spectrum, the placenta forms at a site of disruption between the endometrium and myometrium. Placental tissue implants onto the myometrium (accreta), into the myometrium (increta) or through the myometrium to surrounding organs (percreta) (Figure 1). This prevents placental detachment, which can result in severe hemorrhage and increased risk of maternal morbidity and mortality. ${ }^{1-4}$

2 The most common risk factor is multiple previous cesarean deliveries In a large systematic review, the rate of placenta accreta spectrum increased from $0.3 \%$ in women with 1 previous cesarean delivery to $6.7 \%$ in women with 6 previous cesarean deliveries. ${ }^{5}$ Additional risk factors include uterine surgery and assisted reproduction. ${ }^{1-3}$

\section{3}

\section{Placenta previa substantially increases the risk of placenta accreta spectrum}

Among women with placenta previa and history of previous cesarean delivery, the risk of placenta accreta spectrum increases from $11 \%$ after 1 cesarean delivery to $60 \%$ after 3 or more cesarean deliveries. ${ }^{4,5}$ Placenta previa occurs in about $3 \%$ of pregnancies. ${ }^{5}$

\section{4}

\section{Women with placenta previa and a history of cesarean delivery should have targeted ultrasounds in centres of excellence} Although findings on imaging are present throughout pregnancy, placenta accreta spectrum is commonly suspected during the routine anatomy scan performed at 18 to 20 weeks. ${ }^{1,4} \mathrm{~A}$ combination of transabdominal and transvaginal ultrasound achieves diagnostic sensitivity of 90\% (95\% confidence interval [CI] 87\%-93\%) and specificity of $97 \%$ (95\% Cl 96\%-97\%). ${ }^{1}$ Magnetic resonance imaging can be an adjunct when assessing posterior placentas and depth of invasion. ${ }^{1,4}$

\section{5}

\section{Management of placenta accreta spectrum requires specialized teams}

Early referral to centres of excellence reduces maternal morbidity, including the need for massive transfusion and multiple surgical interventions. ${ }^{1,4}$ Centers of excellence typically follow standardized protocols and care pathways, bringing together specialists in maternal fetal medicine, gynecology, anesthesia and neonatology, as well as allied health professionals.

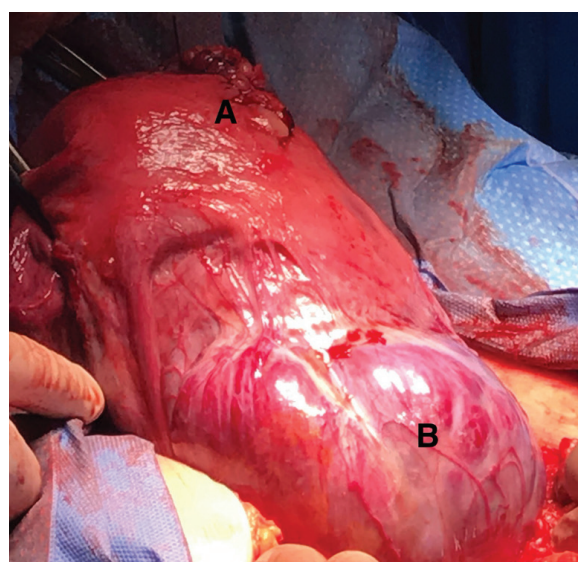

Figure 1: Intraoperative cesarean hysterectomy for placenta percreta in the context of placenta previa. The baby was delivered through a classical fundal incision (A), which was closed before the hysterectomy was begun. The placenta was left in situ with bulging, vascular placental tissue visible under the uterine serosa (B). Final pathology reported placenta percreta with invasion into the bladder wall.

\section{References}

1. Hobson SR, Kingdom JC, Murji A, et al. No. $383-$ Screening, diagnosis, and management of placenta accreta spectrum disorders. J Obstet Gynaecol Can 2019;41:1035-49.

2. Collins SL, Alemdar B, van Beekhuizen HJ, et al.; International Society for Abnormally Invasive Placenta (ISAIP). Evidence-based guidelines for the management of abnormally invasive placenta: recommendations from the International Society for Abnormally Invasive Placenta. Am J Obstet Gynecol 2019;220:511-26.

3. Jauniaux E, Ayres-de-Campos D, Langhoff-Roos J, et al.; FIGO Placenta Accreta Diagnosis and Management Expert Consensus Panel. FIGO classification for the clinical diagnosis of placenta accreta spectrum disorders. Int J Gynaecol Obstet 2019;146:20-4.

4. Obstetric Care Consensus No. 7: Placenta accreta spectrum. Obstet Gynecol 2018;132:e259-75.

5. Marshall NE, Fu R, Guise JM. Impact of multiple cesarean deliveries on maternal morbidity: a systematic review. Am J Obstet Gynecol 2011;205:262.e1-8.
Competing interests: Nicholas Leyland reports receiving research support grants from AbbVie and Allergen, and consulting fees from AbbVie. No other competing interests were declared.

This article has been peer reviewed.
Affiliation: Department of Obstetrics and Gynecology, McMaster University, Hamilton, Ont.

Correspondence to: Nicholas Leyland, leylann@mcmaster.ca 